\section{W. A. Nowlan}

Department of Computer Science University of Manchester Manchester, UK

The pessimists may argue that medicine is now technically and organisationally so large and complex that it cannot be practised effectively by people, and furthermore that health care is becoming unaffordable. This view is extreme but it does reflect the widespread concern over the effectiveness and efficiency of health care services. There is now an established need to ensure that appropriate health care is delivered effectively, and to demonstrate that both human and financial resources are used efficiently. This is just as true for a rural clinic in India as it is for a large hospital complex in North America. Good information systems are considered essential to meeting those needs, and patient record systems are increasingly recognised as the central component of those systems.

The systems presented in this section illustrate the broad range of problems and solutions that can be considered under the general heading of patient record systems. They range from a specialised system for recording detailed immunological data on a selected group of patients, to a large multi-facility system holding medical information on half a million individuals. The motivations behind the development of the systems are also diverse. They include support for dayto-day patient care, assessment of clinical outcomes, clinical research, integration of bibliographic informaHon with patient records, support and

\title{
Synopsis
}

\section{Patient Record Systems}

evaluation of community health programmes, resource allocation, medical audit, improved communications, and many more.

A simple definition of a patient record system is not possible. However, a patient record system is perhaps best characterised by the need to support the care of individual patients based on a detailed representation of the individual's medical record. Such a system is qualitatively different from more general information systems used for health care administration or epidemiological studies on populations of patients. A patient record system must cope with the scale, detail, and complexity of information required for clinical medicine. The long-term vision is of a structured representation of all the information currently found in the paper record, that is suitable for multiple purposes, and amenable to automatic manipulation.

However, at the same time a system needs to be simple and intuitive to use on a routine basis across a range of clinical users and settings. These requirements conflict and present a dilemma to researchers and developers of advanced patient record systems. There is a fundamental trade-off between the detail of the information and the ability to generalise to large-scale systems supporting multiple uses. It is possible to develop a detailed information system for a well-focused medical area, and several successful and useful examples are presented. However, experience has shown it is extremely difficult to scale up such systems or to integrate their functionality and data. At the other end of the spectrum is the twenty years of experience with the Regenstrief system [1]. This is a fascinating account of the considerable and wide range problems to be faced when trying to develop and integrate diverse data sources to form a large-scale patient record system.

The question that inevitably arises is: why is it all so difficult? A short synopsis cannot hope to cover all the issues raised by the papers presented in this section. However, I should like to focus on a couple of key issues that are recurring themes through many of the papers and can be identified as key obstacles to the development, integration, and use of patient record systems. These are the representation of medical concepts and the humancomputer interface.

\section{The Representation of Medical} Concepts, Terminologies, and the Relationship to Medical Records

Each of the systems addresses, either directly or indirectly, the problem of representing in a computer system medical concepts such as 'pneumonia' and 'beta-2-agonist'. The specialised systems have either adopted a preexisting conceptual model (Gerneth et 
al. [2]) or developed one of their own (Bolens et al. [3], Pinciroli et al. [4], Christo et al. [5], Singh et al. [6]). Many of these models have been conceived as a paper pro forma but, nevertheless, they constitute a formal definition and structuring of the pertinent concepts. They are generally modest models of the order of a few hundred data items (concepts) which is a size that a single worker can understand and manage. If interpreted appropriately they can be very effective. Broader systems have employed more widely used terminologies such as DSM-III-R (Powsner and Miller [7]) or SNOMED (Satomura et al. [8]). With this approach compromise is more common and modifications such as those to SNOMED are often necessary to support a specific system (Satomura [8]). It is important to note here that the system for the automatic diagnostic indexing by natural language processing (Satomura [8]) is also critically dependent on the semantics of the domain model, in this case SNOMED. Natural language processing does not remove the need for models of medical concepts, it actually increases that need. The Regenstrief work (McDonald [1]) has faced full on the organisational and technical problems of supporting and reconciling the use of multiple terminologies and classifications. The problems are partly organisational and require people to agree with one other. However, many of the problems are an inevitable consequence of the numerous and often conflicting demands resulting from the multiple uses and sources of information.

In all the systems numerical data are generally handled much better than descriptive information. Despite considerable efforts, the bulk of the patient's clinical record covering for example diseases, symptoms, signs, procedures, and other treatments, cannot yet be represented in a struc- tured form. Most of the information is in the form of free text and the vast majority of this is held on paper records. While it is fair to debate the need for, and feasibility of, a structured representation of the entire medical record, it is clear that the current position is unsatisfactory and progress is urgently needed.

For over 130 years the foundation for the structured recording of medical information has been enumerative classification based on coding schemes such as ICD. This representational approach has been very successful when used appropriately but it is now clearly inadequate when faced with the scale and diversity of patient record systems. The lack of appropriate representational techniques and associated large reusable models of medical concepts is a major obstacle to the development and integration of advanced patient record systems. It remains a hypothesis that such techniques and models are possible, but it must be remembered that the goal is not a representation of the whole of medical knowledge. Quite the contrary. The aim must be to provide a model that is sufficient to support clinical descriptions and discourse. It is vital that the scope is kept realistic. First steps are being taken to address some of these problems with examples in both Europe and North America.

The problem of medical terminologies and concept representation is compounded by our poor understanding of the relationship between terminological models, such as the current coding and classification schemes, and specific information models of the medical record. What should we expect from a comprehensive terminological model and what can be safely or usefully left to the designers of individual information systems? What is the framework for making such decisions? A totally prescriptive approach to the design of patient record models is obvious unacceptable and impossible, but a common core is required if system are to be integrated, communicat and be re-used efficiently. To this must be added the challenge of attending immediate needs while providing for the evolution of systems as improve solutions become available.

\section{The Human-Computer Interface, Data Entry, and Making Systems Acceptable}

McDonald admits that their early perceptions of the problem of the interface and in particular 'data entry' were naive [1]. There are few of us, if any, who could not make the same admission. If the task is seen only as one of data entry then this is usually greeted with little enthusiasm by medical professionals. As a result of this lack of enthusiasm most systems are a blend of direct electronic entry, paper-based collection with data-entry clerks, and a range of other pragmatic solutions. Most of the systems described use paper-based forms or structured dictation as the first stage of data entry, with clerical workers transferring the information to the computer (McDonald [1], Bolens [3], Christo [5], Singh [6], Barrie [9]). It is important to recognise that in most situations the information has already been structured or encoded by the medical professional. This is quite different to the use of coding clerks who have to make a judgement about what information is relevant before coding it. Other systems make use of the dictated 'free' text from case reports and summaries (Satomura [8], Powsner [7]). Only two clearly describe the direct use by medical professionals for data entry (McDonald [1], Pinciroli [4]). McDonald explaing that a paper and person-based frontent to a patient record system can be costeffective for the collection of small 
and well-defined data sets. However, 4 cannot provide direct interaction pith the clinical users and, hence, cannot directly support and influence clinical decision making. It is the provision of information and support to the medical professional that is so important in making patient record systems acceptable and effective. The direct use of systems by medical professionals must remain the goal.

What at first appears odd is that the major advances in user-interface architectures and environments, together with the fall in the real cost of computers and high-quality displays, have not swept away all the difficulties. The reason is that the 'interface' per se is not the problem. The difficulties lie much deeper. Medical professionals require the dialogue with a system to be natural and intuitive. They often feel that the computer system should recognise and display the critical items of information, and make appropriate requests of the user in ways that require a minimal response. However, to achieve this, implies that a system 'understands' the dialogue and the information in the patient record. Few, if any, systems can match these expectations and as a result the dialogue with a system is often intrusive, time consuming, and dominated by the task of 'data entry'. This conjures up a negative and unresponsive image of patient record systems, and fails to engage clinicians.

Why do the interfaces to systems generally fail to meet users' expectations? The reason is partly that the expectations are simply unrealistic given our current abilities and it is important to recognise when that is the case. Medical professionals are generally poor judges of what is technically difficult. However, the failure is also because the interface is the most immediate and obvious victim of the lack of a sound underlying conceptual structure on which to base the behaviour of the system. The problem is similar to many in expert systems work but the task is quite different. The system is not trying to perform a diagnosis or make a therapeutic recommendation. It is trying to respond sensibly to the dialogue. To do so, it needs some understanding of the meaning of a concept within the discourse and this is inextricably bound up with the problem of representing medical concepts. The interface is a stringent test of the underlying medical model. It is thus not possible to treat it as a coat of paint to be applied to the system after the main design has been completed. To the medical user the interface is the system and significant effort is still required to make progress in this area.

Clearly, there are major social and organisational factors that influence the usability and acceptability of patient record systems. In particular there is a need for effective ways of bridging the technical and cultural gaps between clinicians and information scientists. Many of the problems are poorly understood and many will require changes in medical practice as well as technical developments. Many of the accounts presented show that the process may be slow but change is possible if the benefits are clear. The evaluation of large patient record systems is still in its infancy but Barrie [9] shows that it is possible, and gives several important insights into the strong cultural bonds and shared medical models that exist amongst clinicians.

Problems in medical informatics are no different from other medical problems such as the treatment of a major disease group, for example cancer. Magic bullets are few and far between, and most clinical progress is made by learning how best to apply a combination of existing treatments, identi- fying the need for new developments, and incorporating those developments when they become available. Many of the challenges facing the development of patient record systems are about coping with diversity and integrating a range of components. It is inescapable that medicine is large and complicated, and patient record systems are exposed to the full effects of that complexity. However, we need to partition the problem in ways that allow the development of useful and usable systems with realistic intellectual and economic effort.

\section{References}

[1] McDonald CJ, Tierney WM, Overhage JM, Martin DK and Wilson GA. The Regenstrief Medical Record System: 20 years of experience in hospitals, clinics, and neighborhood health centers. MD Comput 1992;9:206-17.

[2] Gerneth F, Haux R and Müller CA. Data modeling for immunological and clinical data of leukemia and myasthenia patients. Meth Inf Med 1992;31:136-46.

[3] Bolens M, Borst F and Scherrer JR. Organizing the clinical data in the medical record. MD Comput 1992;9:149-55.

[4] Pinciroli F, Combi C, Pozzi G and Rossi R. MS2/Cardio: towards a multi-service medical software for cardiology. MethInf Med 1992;31:18-27.

[5] Christo GG, Marianus BVP, Krishnan L, Iyer RS and Venkatesh A. Computerised neonatal case records: a four year experience. Indian Pediatr 1992;29:17380.

[6] Singh AK, Moidu K, Trell E and Wigertz O. Impact on the management and delivery of primary health care by a computerbased information system. Comput Meth Programs Biomed 1992;37:55-64.

[7] Powsner SM and Miller PL. Automated online transition from the medical record to the psychiatric literature. Methods Inf Med 1992;31:169-74.

[8] Satomura $Y$ and do Amaral MB. Automated diagnostic indexing by natural language processing. Med Inf (Lond) 1992;17:149-63.

[9] Barrie JL and Marsh DR. Quality of data in the Manchester orthopaedic database. BMJ 1992;304:159-62. 